\title{
Safety and Efficacy of in Vitro Fertilization in Women with Systemic Lupus Erythematosus and Antiphospholipid Syndrome
}
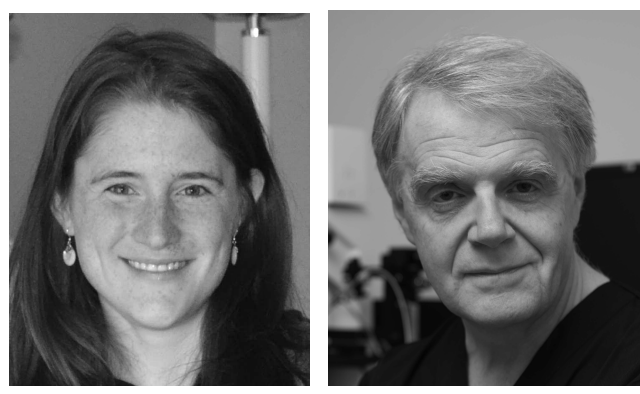

Systemic lupus erythematosus (SLE) is a chronic, multisystem, hormone-sensitive autoimmune disease that can be associated with antiphospholipid syndrome (APS). The prevalence of SLE ranges from 40 to 200 cases per 100,000 persons, depending on ethnic background, and it predominantly affects women of childbearing age. The prognosis of SLE has dramatically improved in recent decades. In the 1950 s, $50 \%$ of patients with SLE survived more than 4 years, and today, the 15 -year survival rate is over $80 \%{ }^{1}$. With improved outcomes, the possibility of conceiving and bearing children is becoming a reality for many women with SLE. Despite increased risks of flares, thrombosis, and gestational complications such as preeclampsia, most women with treated and clinically quiescent SLE can safely carry a pregnancy under appropriate medical supervision ${ }^{2}$.

Infertility rates in SLE, with some exceptions, are similar to those of the general population ${ }^{3}$, and claims that antiphospholipid antibodies (aPL) could contribute to infertility are largely unsubstantiated ${ }^{4}$. Because SLE is an estrogen-sensitive disease, hormonal manipulation carries an inherent risk of SLE flares, hypercoagulability, and ovarian hyperstimulation syndrome (OHSS). Further, the safety of assisted reproductive technology (ART) has not been clearly established in patients with SLE/APS ${ }^{5}$. The relative paucity of data on the subject is explained by the rarity of concomitant SLE and infertility, but with an increasing number of young women achieving disease quiescence, the topic becomes increasingly relevant.

There are rare documented case reports of severe, and 1 fatal SLE/APS exacerbation after ovulation induction $(\mathrm{OI})^{6,7,8}$. However, only 2 series on ART in patients with SLE have been published to date ${ }^{9,10}$.

In this issue of The Journal, Orquevaux, et al retrospectively describe the outcomes of a cohort of 37 SLE and/or APS patients who resorted to in vitro fertilization (IVF) for the treatment of infertility ${ }^{11}$. Ninety-seven IVF procedures, defined as ovulation induction (OI) with oocyte retrieval or an embryo transfer procedure using a donated oocyte were performed in this cohort. Four patients developed SLE flares (3 polyarthritis and 1 lupus enteritis) and 3 patients developed a thromboembolism (1 lumbo-ovarian, 1 distal deep venous, and 1 pulmonary embolism). All complications occurred during ovulation induction protocols using a gonadotropin-releasing hormone $(\mathrm{GnRH})$ agonist, and 4 complications were attributed to noncompliance to treatment. No OHSS was reported. Twenty-seven pregnancies occurred, of which 23 culminated in live births, 2 miscarried, and 2 were electively terminated. The authors conclude that IVF can be safely performed in SLE/APS patients with similar efficacy as in the general population. They caution, however, that patients' disease must be well controlled and monitored, and that adherence to treatment is paramount to maintenance of disease quiescence during ART or pregnancy.

Patients and their physicians must balance the benefit of ART against the risk of thrombosis, disease exacerbation, and OHSS. The difficulty of this task is compounded by the paucity of data on the subject. Currently there are no guidelines or laboratory biomarkers to determine which SLE/APS patient will most likely develop complications from these elective procedures. Orquevaux, et al's study outlines important measures that can be taken to reduce potential risks associated with OI/IVF and significantly contributes to the advancement of our knowledge on the subject.

As in pregnancy, patients with SLE undergoing OI/IVF must be considered at high risk of complications ${ }^{12}$. Extrapolating from known guidelines for pre-pregnancy counseling, the safest time to perform OI/IVF would be once the underlying disease is in remission for at least 6 months or under excellent control on minimal medication; the patient is compliant with therapy; and has discontinued any known teratogenic medication for the recommended washout period

See IVF in SLE and APS, page 613

Personal non-commercial use only. The Journal of Rheumatology Copyright @ 2017 . All rights reserved. 
for each compound. Patients with severe pulmonary hypertension, restrictive lung disease, chronic renal failure, a cerebrovascular event within 6 months, or an obstetric history of severe preeclampsia or HELLP syndrome (hemolysis, elevated liver enzymes, and low platelets) might similarly be at increased risk of complications and should not undergo $\mathrm{OI} / \mathrm{IVF}^{13}$

One of the highlights of Orquevaux's study is that $96 \%$ of the IVF cycles were supervised by an internist who ensured pre-pregnancy counseling and adequate followup during the IVF cycle and pregnancy. As a result, all women undergoing ART were appropriately managed with their underlying disease remaining in clinical remission or very well controlled before undergoing OI/IVF. Close followup during pregnancy ensured the rapid detection and prompt treatment of any SLE flares, minimizing fetal and maternal complications. This complete and comprehensive assessment of patients is paramount and explains the low rate of complications observed in this study.

The superior safety profile of ovarian stimulation protocols using $\mathrm{GnRH}$ antagonists over $\mathrm{GnRH}$ agonists in the general population has been recently shown in a Cochrane metaanalysis update published in $2011^{14}$. IVF cycles managed with GnRH antagonists have reduced peak serum estrogen concentrations and are associated with a $50 \%$ reduction in the relative likelihood of OHSS compared to cycles stimulated with GnRH agonist protocols, and a 10\% lower risk of OHSS when used in women with polycystic ovary syndrome. According to a systematic review of the literature describing thromboembolic complications in relation to ART, 79\% of all thrombotic events occurred in patients with OHSS and 98\% (70/71) of cases appeared after human chorionic gonadotropin (hCG) administration ${ }^{15}$. Applying these data to an SLE population, one can assume that GnRH antagonists would be a safer alternative for OI. Indeed, in Orquevaux's study, GnRH agonist protocols were used in $51.5 \%$ of cycles, antagonist protocols in $15.5 \%$ of procedures, and natural cycles were used for retrieval in $25 \%$. Interestingly, all complications observed in this study occurred in cycles that included GnRH agonists for ovulation induction. No complications were observed in GnRH antagonist protocols or natural cycles, emphasizing that less aggressive ovarian stimulation, which avoids high serum estrogen concentrations, is preferable in SLE/APS patients.

Surprisingly, no cases of OHSS were reported in the Orquevaux study. The use of GnRH antagonists (thus avoiding the use of exogenous hCG to trigger ovulation) in $15 \%$ of cycles might partially explain this observation. However, other methods were used to minimize the risk for OHSS including hCG injection cancellation with high serum estradiol levels or large number of oocytes, leading to cycle cancellation and oocyte freezing. This appropriately conservative approach to ovarian stimulation in SLE/APS patients should be applied in all cases.
The authors did not comment on any disease exacerbation or thromboembolic events associated with the use of oral estradiol (exogenous estrogen) during donor oocyte cycles where the patient did not undergo ovarian stimulation but merely embryo transfer. No complications occurred in such patients. However, it is important to note that this does represent very different patient treatment and is not directly comparable to those undergoing an IVF cycle involving ovarian stimulation. Without ovarian stimulation, OHSS simply does not occur.

According to the authors, the $28 \%$ IVF pregnancy rate and $85 \%$ live birth rate in their cohort is comparable to that of the general population. They did not find any correlation between APS or aPL and the IVF pregnancy rate. This reinforces the belief that SLE/APS is not associated with subfertility and lower outcomes in ART cycles.

Despite the limitations of the study including small sample size, retrospective design, the unavailable documentation of $8 \%$ of the ovulation induction protocols, and the inclusion of oocyte donation protocols, this remains the most comprehensive and complete study available to date. Several lessons can be learned from Orquevaux, et al's work. Importantly, IVF protocols can be safely and effectively carried out in appropriately selected SLE/APS patients. These patients must be assessed prior to ART treatment and confirmed to be in clinical remission or well controlled on pregnancy-safe medication. They should receive appropriate counseling regarding the ART treatment as well as pre-pregnancy counseling. Ongoing monitoring and followup with an experienced internist/rheumatologist will minimize the risk of adverse events, and if such occur, it is more likely that they will be managed appropriately. Avoidance of OHSS is of paramount importance to reduce the potential for thrombosis and disease flares. Lastly, a conservative approach to ovarian stimulation should be strongly encouraged in this population.

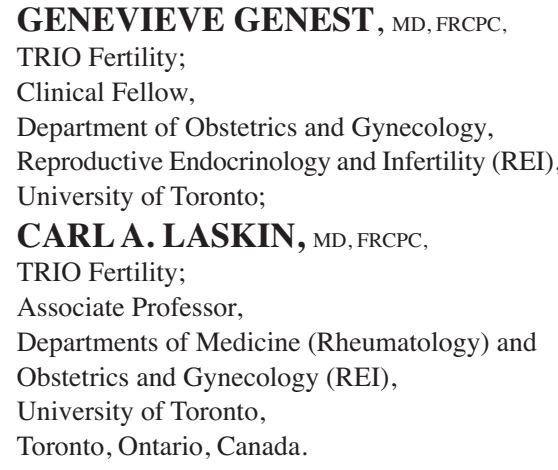

Address correspondence to Dr. C.A. Laskin, TRIO Fertility, 655 Bay St., 18th Floor, Toronto, Ontario M5G 2K4, Canada. E-mail: calaskin@gmail.com

\section{REFERENCES}

1. Rahman A, Isenberg DA. Systemic lupus erythematosus. N Engl J Med 2008;358:929-39.

Personal non-commercial use only. The Journal of Rheumatology Copyright @ ${ }^{2017}$. All rights reserved. 
2. Moroni G, Ponticelli C. Pregnancy in women with systemic lupus erythematosus (SLE). Eur J Int Med 2016;32:7-12.

3. Lockshin MD. Autoimmunity, infertility and assisted reproductive technologies. Lupus 2004;13:669-72.

4. Chighizola CB, de Jesus GR. Antiphospholipid antibodies and infertility. Lupus 2014;23:1232-8.

5. Orquevaux P, Masseau A, Le Guern V, Gayet V, Vauthier D, Boutin $\mathrm{D}$, et al. [In vitro fertilization and systemic lupus erythematosus or antiphospholipid syndrome: an update]. [Article in French] Rev Med Interne 2015;36:154-8.

6. Koo EJ, Rha JH, Lee BI, Kim MO, Ha CK. A case of cerebral infarct in combined antiphospholipid antibody and ovarian hyperstimulation syndrome. J Korean Med Sci 2002;17:574-6.

7. Giner V, Oltra MR, Esteban MJ, Garca-Fuster MJ, Salvador A, Nunez J, et al. Catastrophic antiphospholipid syndrome related to severe ovarian hyperstimulation. Clin Rheumatol 2007;26:991-3.

8. Casoli P, Tuminati B, La Sala G. Fatal exacerbation of systemic lupus erythematosus after induction of ovulation. J Rheumatol 1997;24:1639-40.

9. Guballa N, Sammaritano L, Schwartzman S, Buyon J, Lockshin MD. Ovulation induction and in-vitro fertilization in systemic lupus erythematosus and antiphospholipid syndrome. Arthritis Rheum 2000;43:550-6.

10. Huong DL, Wechsler B, Vauthier-Brouzes D, Duhaut P, Costedoat $\mathrm{N}$, Lefebvre $\mathrm{G}$, et al. Importance of planning ovulation induction therapy in systemic lupus erythematosus and antiphospholipid syndrome: a single center retrospective study of 21 cases and 114 cycles. Semin Arthritis Rheum 2002;32:174-88.

11. Orquevaux P, Masseau A, Le Guern V, Gayet V, Vauthier D, Guettrot-Imbert G, et al. In vitro fertilization in 37 women with systemic lupus erythematosus or antiphospholipid syndrome: a series of 97 procedures. J Rheumatol 2017;44:613-8.

12. Bellver J, Pellicer A. Ovarian stimulation for ovulation induction and in vitro fertilization in patients with systemic lupus erythematosus and antiphospholipid syndrome. Fertil Steril 2009; 92:1803-10.

13. Saavedra Salinas MÁ, Barrera Cruz A, Cabral Castaneda AR, Jara Quezada LJ, Arce-Salinas CA, Álvarez Nemegyei J, et al. Clinical practice guidelines for the management of pregnancy in women with autoimmune rheumatic diseases of the Mexican College of Rheumatology. Part I. Reumatol Clin 2015;11:295-304.

14. Al-Inany HG, Youssef MA, Aboulghar M, Broekmans F, Sterrenburg M, Smit J, et al. GnRH antagonists are safer than agonists: an update of a Cochrane review. Hum Reprod Update 2011;17:435.

15. Chan WS, Dixon ME. The "ART" of thromboembolism: a review of assisted reproductive technology and thromboembolic complications. Thromb Res 2008;121:713-26.

J Rheumatol 2017;44:547-9; doi:10.3899/jrheum.170064 